\title{
Effect of Warm to Hot Rolling on Microstructure, Texture and Mechanical Properties of an Advanced Medium-Mn Steel
}

\author{
MARINA TIKHONOVA, VLADIMIR TORGANCHUK, FREDERIKE BRASCHE, \\ DMITRI A. MOLODOV, ANDREY BELYAKOV, and RUSTAM KAIBYSHEV
}

\begin{abstract}
The deformation microstructures and mechanical properties were studied in a medium-Mn austenitic steel subjected to warm-to-hot rolling. During warm rolling at temperatures of $T<1073 \mathrm{~K}$ the structural changes were controlled by dynamic and static recovery leading to a pancaked work-hardened microstructure, during hot rolling at $T \geq 1073 \mathrm{~K}$-by discontinuous dynamic and post-dynamic recrystallization resulting in equiaxed grains. The grain size decreased while the dislocation density increased with a decrease in rolling temperature. A decrease in rolling temperature enhanced the texture development, which consisted of relatively strong Brass, $\mathrm{S}$ and $\mathrm{P}$ components. The Brass component exhibited the strongest temperature dependence. A decrease in rolling temperature resulted in significant strengthening of the steel. The yield strength increased from 340 to $950 \mathrm{MPa}$ as rolling temperature decreased from $1373 \mathrm{~K}$ to $773 \mathrm{~K}$. Both the grain refinement and the work-hardening contributed to the strengthening.
\end{abstract}

https://doi.org/10.1007/s11661-019-05340-8

(c) The Minerals, Metals \& Materials Society and ASM International 2019

\section{INTRODUCTION}

HiGH-MN structural steels have aroused a great interest among material scientists and mechanical engineers. ${ }^{[1-3]}$ Such special attention has been caused by a beneficial combination of mechanical properties of these materials, namely high strength along with high ductility, owing to so-called twinning-induced plasticity (TWIP) or transformation-induced plasticity (TRIP) effects. The latter is associated with $\varepsilon$-martensitic transformation. The TWIP effect in austenitic steels can be expected, when the stacking fault energy (SFE) value lies between about 20 to $50 \mathrm{~mJ} / \mathrm{m}^{2}$, whereas the TRIP effect occurs at lower SFE values. ${ }^{[4]}$ An appropriate value of SFE is commonly achieved by alloying with a high amount of manganese (18 to $30 \mathrm{pct}$ ), and carbon ( 0.2 to $0.6 \mathrm{pct}) .{ }^{[4,5]}$ Aluminum and silicon are also sometimes used as alloying supplements for high-Mn austenitic TWIP steels to adjust SFE, provide desirable solution strengthening, and suppress delayed fracture. ${ }^{[6-8]}$ The alloying design that involves high manganese percentage

MARINA TIKHONOVA, VLADIMIR TORGANCHUK, ANDREY BELYAKOV, and RUSTAM KAIBYSHEV are with the Belgorod State University, Belgorod 308015, Russia. Contact e-mail: belyakov@bsu.edu.ru FREDERIKE BRASCHE and DMITRI A. MOLODOV are with the Institute of Physical Metallurgy and Metal Physics, RWTH Aachen University, Aachen 52074, Germany.

Manuscript submitted February 5, 2019.

Article published online July 2, 2019 causes a relatively high cost of the steel production that is one of the main disadvantages of high-Mn TWIP steels.

Currently, medium-Mn steels are considered as an alternative to high-Mn steels. ${ }^{[9]}$ They are relatively cheap and can offer an improved combination of mechanical properties under certain conditions. ${ }^{[10-13]}$ It is generally agreed that both high strength and plasticity in high-to-medium-Mn steels is governed by the austenitic phase, which exhibits TWIP or TRIP effects depending on SFE and austenite stability. ${ }^{[9,14,15]}$ Thus, an increase in austenite fraction should be favorable for the mechanical properties of medium-Mn steels. On the other hand, the austenite should have an appropriate SFE and should be rather stable against $\alpha^{\prime}$-martensitic transformation, which is much less effective for the strain hardening than $\varepsilon$-martensitic transformation. In the case of medium-Mn steel containing 5 to 10 pct Mn, enhanced mechanical properties are achieved by intercritical annealing in the two phase (austenite-ferrite) region, when the redistribution of alloying elements (mainly carbon) provides an appropriate combination of austenite fraction and properties. ${ }^{[16-18]}$ Both the fraction and stability of austenite increase with an increase in manganese and carbon content. Almost single phase austenitic steel can be obtained by alloying with $12 \mathrm{pct}$ $\mathrm{Mn}$ and $0.6 \mathrm{pct} \mathrm{C}^{\left[{ }^{[19]}\right.}$ Such steel, i.e., Fe-12 pct Mn-0.6 pct C, should combine a relatively low price of medium-Mn two-phase austenitic-ferritic steels and 
improved mechanical properties of high-Mn austenitic steels. This price-property compromise advances the Fe-12 pct Mn-0.6 pet $\mathrm{C}$ steel as a promising structural material for various engineering applications. The Fe-12 pct Mn-0.6 pct C medium-Mn austenitic steel can be processed by conventional thermo-mechanical treatment including hot rolling without any intercritical annealing. In this case, the final microstructure and mechanical properties of the steel should depend mainly on the rolling temperature. In contrast to high-Mn austenitic steels, however, the structure to property relationships in medium-Mn austenitic steels subjected to warm-to-hot rolling have not been studied systematically. Single-phase medium-Mn austenitic steels with high carbon content above 0.7 pct are not treated in the range of around 0.5 to $0.6 \mathrm{Tm}$ (melting temperature) to avoid the cementite precipitation at grain boundaries. ${ }^{[2]}$ On the other hand, the most of research works on medium-Mn steels with relatively low carbon contents have been focused on the effect of intercritical annealing conditions on the properties of multiphase (i.e., austenite-ferrite-martensite) microstructures. ${ }^{[9]}$

The aim of the present study is to clarify the effect of rolling temperature in the range of $773 \mathrm{~K}$ to $1373 \mathrm{~K}$ on the developed deformation microstructures and textures and their effect on the mechanical properties of an Fe-12 pct Mn-0.6 pct C type steel. Specific attention is paid for the relationship between the mechanisms of microstructure and texture evolution during warmto-hot rolling.

\section{EXPERIMENTAL}

The steel, Fe-12.4Mn-0.57C-1.5Al-0.4Si-0.02Cr-0.03P (all in wt pct), was studied. The steel was produced by induction melting and, then, hot rolling at $1423 \mathrm{~K}$ with 60 pct thickness reduction (from 50 to $20 \mathrm{~mm}$ ). The starting material (after hot rolling at $1423 \mathrm{~K}$ ) was characterized by a uniform microstructure consisting of equiaxed grains with an average size of $80 \pm 8 \mu \mathrm{m}$ with a weak fiber texture of $\langle 100\rangle$ along the normal direction (ND) as shown in Figure 1. The SFE value was roughly evaluated to be $20 \mathrm{~mJ} / \mathrm{m}^{2}{ }^{[4]}$ The steel plates were subjected to rolling at various temperatures from $773 \mathrm{~K}$ to $1373 \mathrm{~K}$ to a total rolling reduction of $60 \mathrm{pct}$ (from 20 to $8 \mathrm{~mm}$ ) followed by air cooling. After each rolling pass with about 10 pct reduction, the samples were re-heated for 4 to 10 minutes to the designated rolling temperature.

The structural investigations were carried out on the sample section normal to the transverse direction (TD) using a Quanta Nova NanoSEM 450 scanning electron microscope (SEM) equipped with an electron back scattering diffraction pattern (EBSD) analyzer incorporating an orientation imaging microscopy (OIM) system. The specimens for structural observations were electro-polished using a 10 pct perchloric acid in acetic acid at a voltage of $20 \mathrm{~V}$ at room temperature. The OIM micrographs were obtained for areas of $0.6 \mathrm{~mm} \times 0.6 \mathrm{~mm}$ with a step of $1 \mu \mathrm{m}$. The EBSD patterns with confidence index below 0.1 were omitted from the OIM analysis (these data-points are filled in black in the OIM images). The OIM software (TSL OIM Analysis 6.2) was used for evaluation of the mean grain size $(D)$ and kernel average misorientation (KAM). The grain size was evaluated by a linear intercept along ND, counting all boundaries with misorientation of $\theta \geq 15 \mathrm{deg}$. The twin boundaries were omitted from the grain size calculations for the recrystallization analysis, whereas the strengthening was analyzed using the grain size including the twin boundaries. KAM was utilized to calculate the dislocation density, $\rho=2(\mathrm{KAM}) /(\mathrm{b} h)$, where $\mathrm{b}$ is the Burgers vector and $h$ is the distance between the measured points in OIM. ${ }^{[20]}$ The rolling textures were studied using a Bruker D8 Advance diffractometer with a HI-STAR area detector and polycapillary focusing optics. The orientation distribution functions (ODF) were calculated in MatLab with the MTEX toolbox ${ }^{[21]}$ using the data from three incomplete pole figures, i.e. $\{111\}$, $\{200\}$, and $\{220\}$. Assuming a spread of $15 \mathrm{deg}$ from the respective ideal orientation, the volume fractions of the various texture components were calculated. The tensile
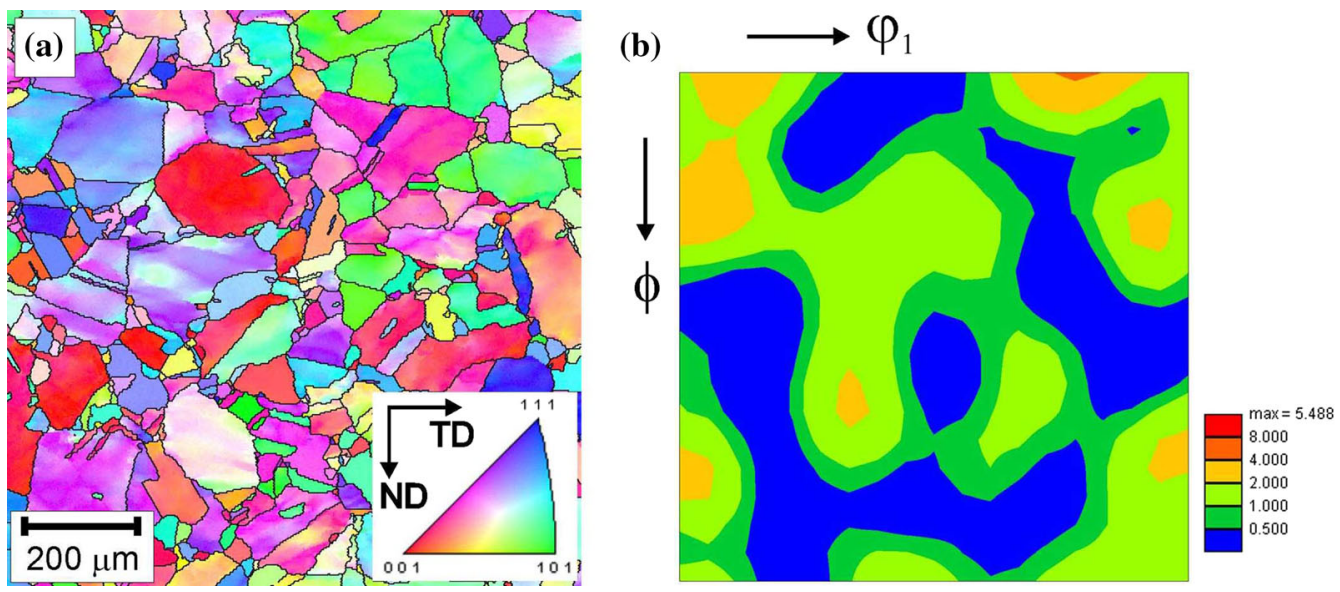

Fig. 1-Initial microstructure $(a)$ and corresponding orientation distribution functions at $\varphi_{2}=45 \mathrm{deg}(b)$ of a medium-Mn steel after hot rolling at $1423 \mathrm{~K}$. Colors indicate the crystallographic direction along ND (Color figure online). 
tests were performed along the rolling direction at ambient temperature under a strain rate of $10^{-3} \mathrm{~s}^{-1}$ using an Instron 5882 on specimens with a gauge length of $12 \mathrm{~mm}$ and a cross-section of $1.5 \mathrm{~mm} \times 3 \mathrm{~mm}$.

\section{RESULTS}

\section{A. Warm-to-Hot Worked Microstructures}

Typical deformation microstructures are shown in Figure 2. Two deformation temperature domains corresponding to warm or hot working conditions can be distinguished in Figure 2. Warm rolling at temperatures of $773 \mathrm{~K}$ to $973 \mathrm{~K}$ results in the elongation of original grains along the rolling direction. In contrast, hot rolling at temperatures of $1073 \mathrm{~K}$ to $1373 \mathrm{~K}$ leads to the development of equiaxed grains. The remnants of original grains can be observed in Figure 2(c) as highly elongated grains. Setting the minimal aspect ratio (the length of the minor axis divided by the length of the major axis) for recrystallized grains as 0.25 , the recrystallized fraction after rolling at $1073 \mathrm{~K}$ comprises 0.85 (Figure 2(c)). An increase in rolling temperature leads to fully recrystallized microstructure. The grains evolved by hot-rolling contain numerous annealing twins and
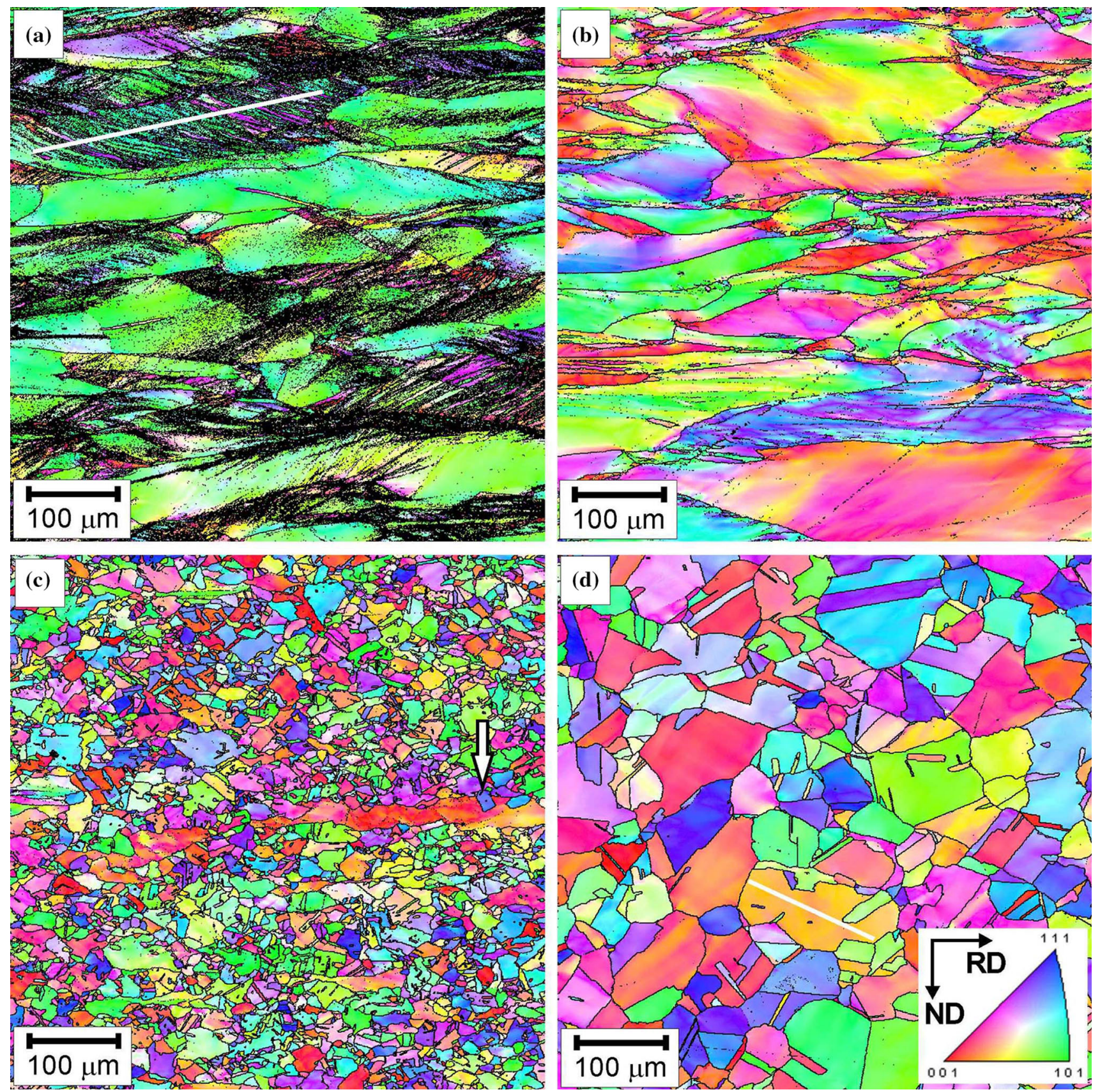

Fig. 2-Typical OIM images of deformation microstructures evolved in a medium-Mn steel subjected to rolling at temperatures of $773 \mathrm{~K}$ (a), $973 \mathrm{~K}(b), 1073 \mathrm{~K}(c), 1273 \mathrm{~K}(d)$. The colors indicate the direction along ND (Color figure online). 
the mean grain size decreases with a decrease in the deformation temperature. It can be concluded, therefore, that the microstructure evolution in the steel samples subjected to hot rolling results from discontinuous dynamic recrystallization including post-dynamic recrystallization, involving grain boundary bulging as the mechanism of grain nucleation. An example of new grain development by the bulging mechanism is indicated by the white arrow in Figure 2(c). This is frequently observed mechanism of microstructure evolution in low-to-medium SFE metals and alloys processed by large strain hot working. ${ }^{[22-25]}$

The flattened grains in the steel sample, which was warm rolled at $773 \mathrm{~K}$, frequently contain a number of closely spaced boundaries appearing as dark bands on the OIM micrograph (Figure 2(a)). The change in the crystallographic orientation along the white line in Figure 2(a) is represented in Figure 3(a) as point-to-origin misorientation. It is clearly seen in Figure 3(a) that the orientation frequently varies over $60 \mathrm{deg}$ as the measuring point moves along the line. This change in orientation by $60 \mathrm{deg}$ and backward testifies to deformation twinning, which operated upon warm rolling. Deformation twinning is a common deformation

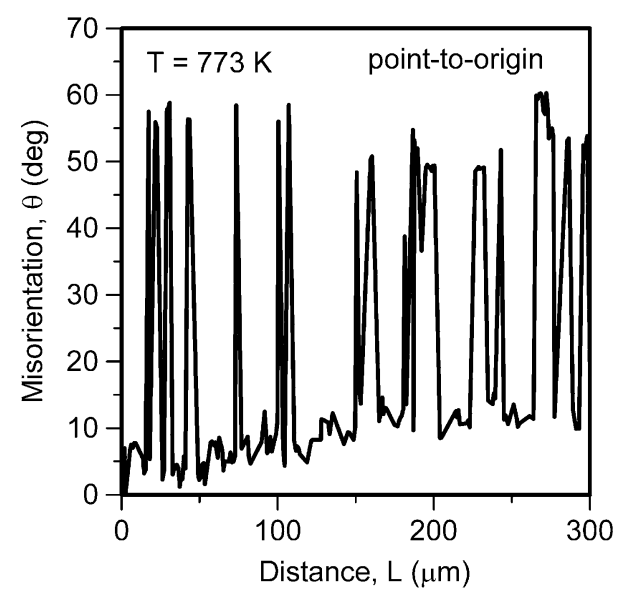

(a)

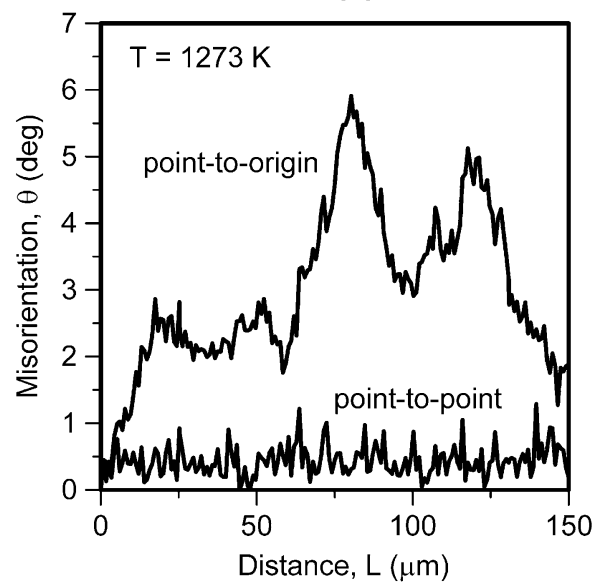

(b)

Fig. 3-Misorientations within the grains evolved during rolling at $773 \mathrm{~K}(\mathrm{a})$ and $1273 \mathrm{~K}(\mathrm{~b})$ along the lines indicated in Figs. 2(a) and (d), respectively. mechanism acting in low SFE fcc-metals and alloys during cold deformation. ${ }^{[26,27]}$ An increase in deformation temperature increases the SFE value and, therefore, should suppress deformation twinning. ${ }^{[28,29]}$ The obtained results indicate that deformation twinning can occur in the present steel during rolling even at such a high temperature of $773 \mathrm{~K}$. It should be noted that the flattened and twinned grains are characterized by large internal distortions after warm rolling. The cumulative misorientation within an individual grain, e.g. the matrix of the grain marked by the white line in Figure 2(a), attains about $10 \mathrm{deg}$ (Figure 3(a)). This is indicative of high dislocation densities that evolve in the steel samples and strengthen them during warm rolling.

The steel samples subjected to hot rolling are also characterized by high residual stresses. The change in the orientation along the white line indicated in Figure 2(d) is represented in Figure 3(b). The lattice curvature over the grain (point-to-origin) achieves $6 \mathrm{deg}$, although the misorientation between any neighboring points (point-to-point) does not exceed $1 \mathrm{deg}$. The selected grain in Figure 2(d) contains annealing twins that suggests its discontinuous recrystallization origin, i.e., nucleation followed by growth in course of dynamic or post-dynamic recrystallization. The large internal distortions as shown in Figure 3(b) testify to rather high dislocation densities evolved in the steel samples subjected to hot rolling irrespective of discontinuous recrystallization taking place during and/or after deformation. Thus, the dislocation strengthening should be taken into account while predicting the strengthening by warm-to-hot working.

The temperature effect on hardness $(\mathrm{Hv})$, the grain size $(D)$ and the kernel average misorientation (KAM), which roughly corresponds to internal distortion, are shown in Figure 4. The grain sizes shown by open symbols in Figure 4 were calculated including the twin boundaries, whereas those indicated by solid symbols were evaluated without counting the twin boundaries. Generally, $D$ increases while $\mathrm{Hv}$ and KAM decrease with an increase in the rolling temperature. The change

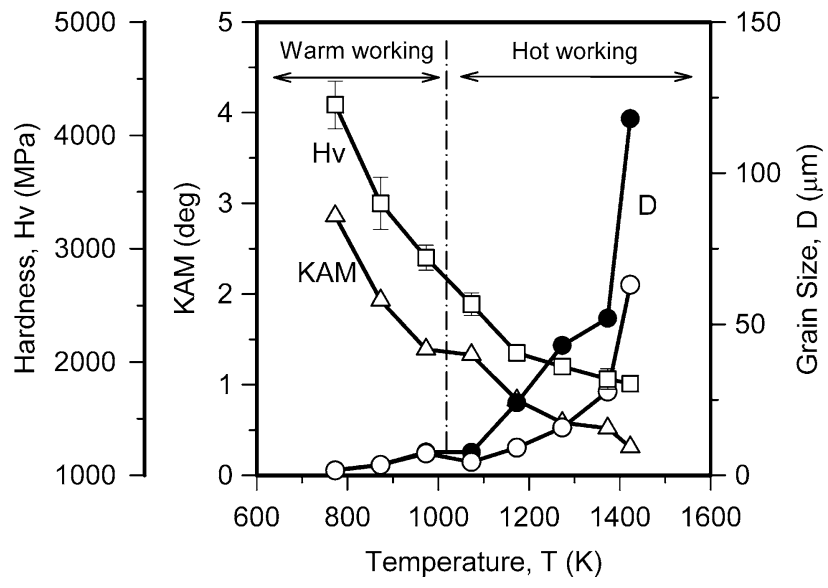

Fig. 4-Effect of rolling temperature on hardness (Hv), average grain size $(D)$ and kernel average misorientation (KAM) in a medium-Mn steel. The twin boundaries were included (open symbols) or excluded (solid symbols) from the grain size calculation. 
in the deformation behavior that is associated with a transition from warm to hot working is reflected by rather smooth D-T and KAM-T plots as small kinks around $1000 \mathrm{~K}$ in Figure 4, although Hv does not show any significant inflections at this temperature. The grain size developed during hot working through dynamic and post-dynamic recrystallization, depends on grain boundary migration ability and, thus, decreases with a decrease in deformation temperature. ${ }^{[30]}$ In the case of warm working accompanied by dynamic recovery only, the grain size, which is measured as a high-angle boundary spacing in the normal direction, depends on the rolling reduction and the number of strain induced grain boundaries developed during deformation. The decreasing grain size in the range of $773 \mathrm{~K}$ to $973 \mathrm{~K}$ in Figure 4 suggests that the number of strain induced grain boundaries increases with a decrease in deformation temperature under warm working conditions. It should be noted that the deformation twinning at relatively low temperatures also contributes to the grain size reduction. Slowing down the recrystallization kinetics and the recovery processes with a decrease in the deformation temperature results in gradually increasing the internal distortions and related KAM. It is worth noting that the recrystallization cessation with a decrease in deformation temperature affects the recovery of internal distortions that leads to specific jump in the temperature-KAM dependence (Figure 4).

The different mechanisms of microstructure evolution during warm and hot rolling lead to characteristic grain boundary assemblies that develop in either work-hardened or recrystallized microstructures (Figure 5). The work-hardened microstructures that developed during warm rolling at temperatures of $773 \mathrm{~K}$ to $973 \mathrm{~K}$ are characterized by a sharp peak against misorientations below 6 deg in Figure 5. Correspondingly, the fraction of low-angle subboundaries with misorientations of $\theta<15 \mathrm{deg}\left(F_{\mathrm{LAB}}\right.$ in Figure 5$)$ may exceed 0.9 in the warm rolled samples. In contrast, the dynamically and/ or post dynamically recrystallized microstructures in the samples hot rolled at temperatures above $1073 \mathrm{~K}$ involve large fractions of CSL $\Sigma 3$ boundaries $\left(\mathrm{F}_{\Sigma 3}\right.$ in Figure 5) corresponding to annealing twins. Note here that $\mathrm{F}_{\Sigma 3}$ shown in Figure 5 were obtained by calculation among high-angle boundaries only, i.e., low-angle subboundaries were not taken into account while evaluating the fractions of special CSL $\Sigma 3$ boundaries. Therefore, the grain boundary misorientation distribution in the dynamically and/or post-dynamically recrystallized microstructures exhibit two sharp peaks corresponding to low-angle subboundaries $(\theta<15 \mathrm{deg})$ and twin boundaries $(\theta>55 \mathrm{deg})$, whereas other misorientations between these two peaks are characterized by near random distribution. The latter ${ }^{[31]}$ is indicated by the dashed line in Figure 5.

\section{B. Deformation Textures}

The representative sections of orientation distribution functions (ODF) at $\varphi_{2}=45 \mathrm{deg}$ for the samples subjected to warm-to-hot rolling are shown in Figure 6. The main texture components, which are typical for low SFE austenite are represented in Figure 7 and defined in Table I. The deformation textures are characterized by the development of a rather strong $\alpha$-fiber with a maximum at around Brass component and a somewhat

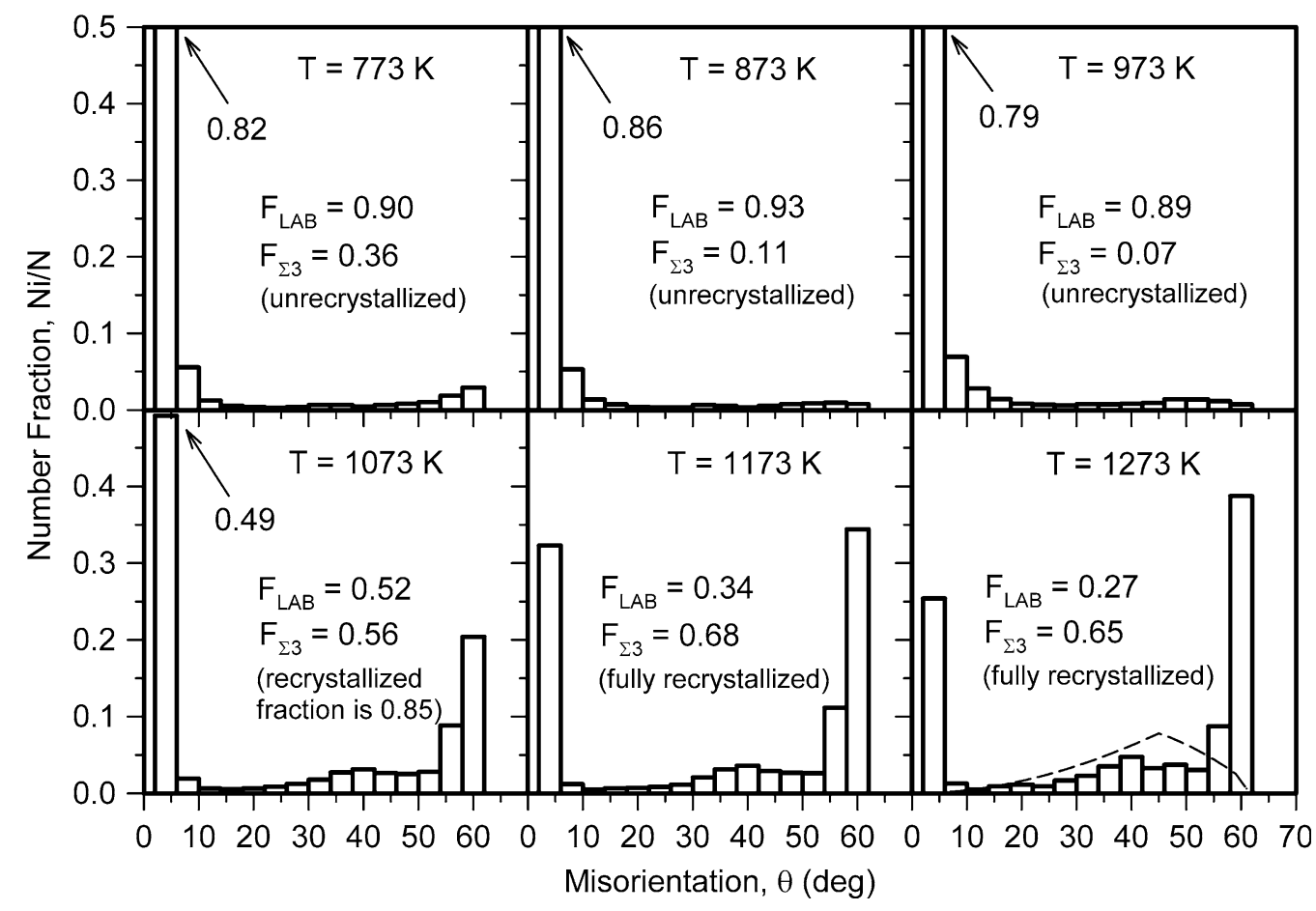

Fig. 5-Grain boundary misorientation distributions for a medium-Mn steel subjected to warm-to-hot rolling at indicated temperatures. The random misorientation distribution ${ }^{[31]}$ is indicated by the dashed line. 


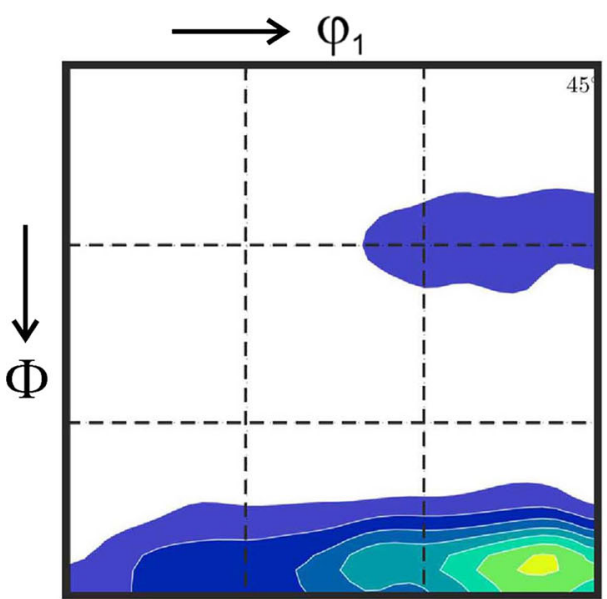

(a)

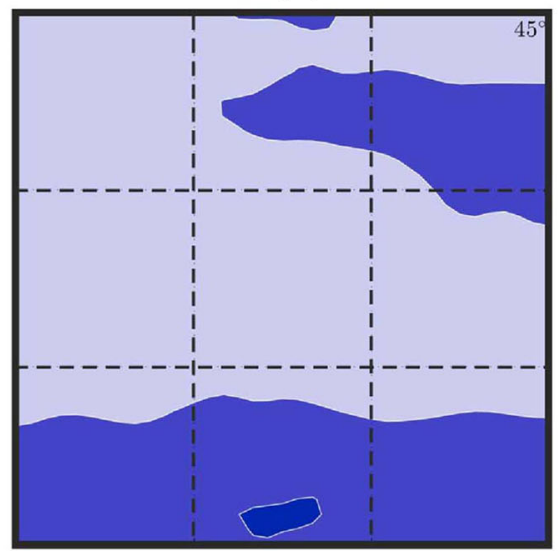

(c)

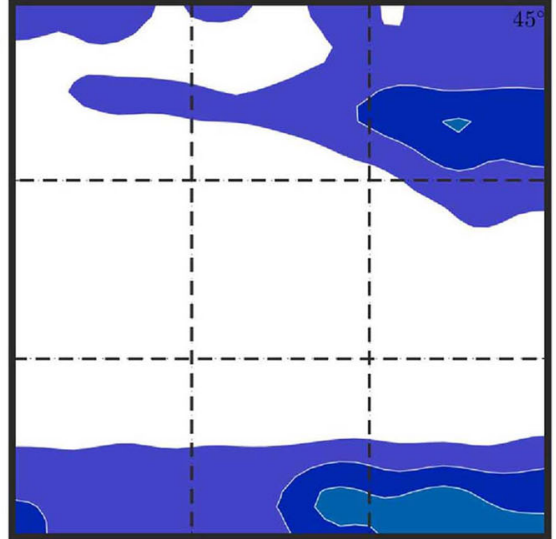

(b)

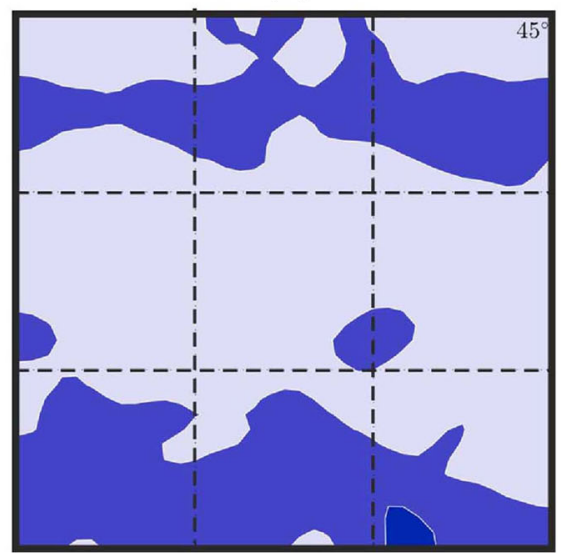

(d)

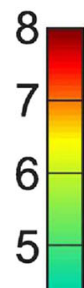

4

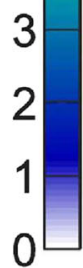

Fig. 6 - Orientation distribution functions at $\varphi_{2}=45 \mathrm{deg}$ of a medium-Mn steel subjected to rolling at temperatures of $773 \mathrm{~K}(a)$, $973 \mathrm{~K}(b)$, $1173 \mathrm{~K}(c)$, and $1373 \mathrm{~K}(d)$.

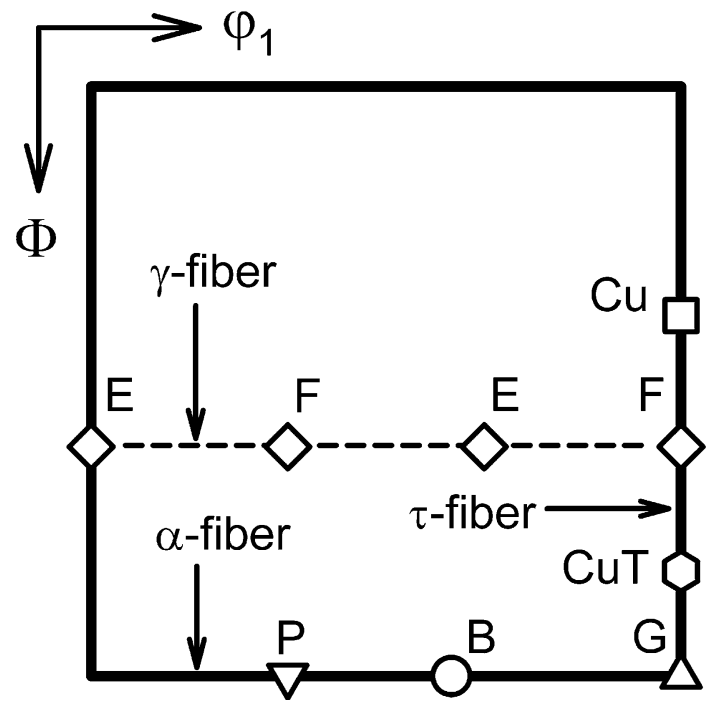

Fig. 7-Typical texture components at $\varphi_{2}=45 \mathrm{deg}$ in a low SFE austenite subjected to plate rolling.

well-built maximum, which looks like $\mathrm{Cu}$ component shifted to $\varphi_{1}=90 \mathrm{deg}, \varphi=20 \mathrm{deg}, \varphi_{2}=45 \mathrm{deg}$ corresponding to $\{114\}\langle 221\rangle$. The latter gradually attenuates with decreasing $\varphi_{1}$ (Figure 6). The development of $\alpha$-fiber and Brass component has been frequently observed in high-Mn austenitic steels with low SFE subjected to cold rolling. ${ }^{[32-35]}$ On the other hand, cold rolling has not been accompanied by the texture development around $\{114\}\langle 221\rangle$. Moreover, the present steels processed by warm-to-hot rolling do not exhibit remarkable $\gamma$-fiber, which has been reported for high-Mn steels after large strain cold rolling. ${ }^{[33,35]}$

Generally, the texture intensity decreases with an increase in deformation temperature (Figure 8). The steel samples exhibit notable Brass, S, P, and Goss components after warm rolling at $773 \mathrm{~K}$. The Brass and Goss components demonstrate the most remarkable reduction; their fractions decrease more than twofold with an increase in the rolling temperature from $773 \mathrm{~K}$ to $1373 \mathrm{~K}$. The $\mathrm{S}$ and $\mathrm{P}$ components also weaken with increasing temperature, although the $\mathrm{S}$ component remains one of the most intensive compared to other components irrespective of deformation temperature. As also seen in Figure 8, the fraction of Copper Twin (CuT) component does not remarkably alter from random orientation conditions for samples rolled at different temperatures; it slightly varies around 0.03 irrespective of deformation twinning during warm 
Table I. Definition of Texture Component

\begin{tabular}{llcl}
\hline Component & Miller Indices & Euler Angles (Deg) & Fiber \\
\hline Brass (B) & $\{110\}\langle 112\rangle$ & $55,90,45$ & $\alpha, \beta$ \\
Goss (G) & $\{110\}\langle 001\rangle$ & $90,90,45$ & $0,90,45$ \\
Rotated Goss (RtG) & $\{110\}\langle 110\rangle$ & $90,35,45$ & $\alpha$ \\
Copper (Cu) & $\{112\}\langle 111\rangle$ & $90,74,45$ & \\
Copper Twin (CuT) & $\{552\}\langle 115\rangle$ & $0 / 60,55,45$ & $30 / 90,55,45$ \\
E & $\{111\}\langle 110\rangle$ & $20,90,45$ & \\
F & $\{111\}\langle 112\rangle$ & $59,37,63$ & $\gamma$ \\
P & $\{110\}\langle 122\rangle$ & & \\
S & $\{123\}\langle 634\rangle$ & & \\
$\alpha$-Fiber & $\langle 110\rangle$ Parallel to ND & & \\
$\gamma$-Fiber & $\langle 111\rangle$ Parallel to ND & & \\
$\tau$-Fiber & $\langle 110\rangle$ Parallel to TD & & \\
\hline
\end{tabular}

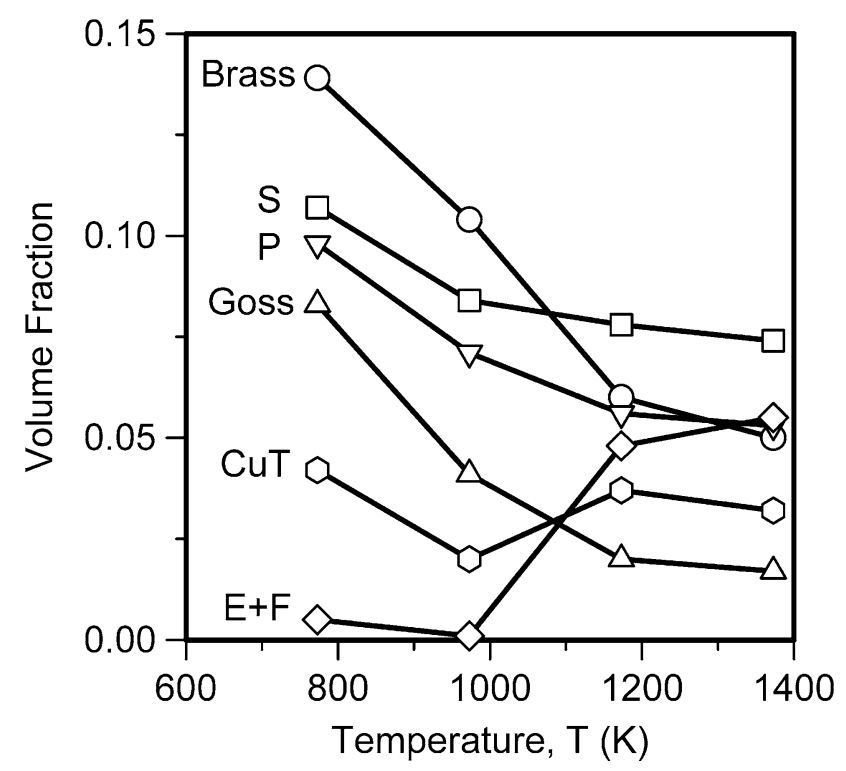

Fig. 8-Temperature effect on the volume fractions of texture components in a medium-Mn steel processed by warm-to-hot rolling.

rolling and formation of annealing twins upon hot rolling. On the other hand, the fraction of $\mathrm{E}+\mathrm{F}$ components increases from almost zero to about 0.05 with an increase in the rolling temperature from warm to hot working domain.

The difference in the texture development during warm-to-hot rolling from ordinary cold rolling can be associated with an increase in SFE as temperature increases. Indeed, the fractions of texture components like Brass, S, Goss etc., which are typical for cold rolled austenite, increase with a decrease in rolling temperature. Regarding the $\mathrm{E}+\mathrm{F}$ components, their development during cold rolling has been attributed to shear banding at large strains. ${ }^{[35]}$ The present warm-to-hot rolling is not accompanied by shear banding. Correspondingly, the fraction of $\mathrm{E}+\mathrm{F}$ components should be negligibly small. An apparent increase in the fraction of $\mathrm{E}+\mathrm{F}$ components may be associated with an overall texture randomization caused by dynamic and post-dynamic recrystallization. The effect of rolling temperature on the $\gamma$-fiber development can be explained in a similar way. Namely, the texture randomization upon increasing temperature is accompanied by an apparent increase in the orientation density along $\gamma$-fiber (Figure 6).

\section{Tensile Tests}

The stress-strain curves obtained by tensile tests of the present warm-to-hot rolled steel samples are shown in Figure 9; the tensile properties are collected in Table II. A decrease in rolling temperature leads to significant strengthening. It is worth noting that the effect of rolling temperature on the yield strength $\left(\sigma_{0.2}\right)$ is much more pronounced than that on the ultimate tensile strength (UTS). The former increases from 360 to $950 \mathrm{MPa}$ while the latter increases from 1100 to $1300 \mathrm{MPa}$ with a decrease in the rolling temperature from $1373 \mathrm{~K}$ to $773 \mathrm{~K}$ (Table II). The strengthening by warm rolling is accompanied by a decrease in plasticity, although rather large uniform elongation above 15 pct remains even after rolling at the lowest applied temperature of $773 \mathrm{~K}$. The strength properties, i.e., the ranges of $\sigma_{0.2}$ and UTS obtained in the present warm to hot rolled steel samples, are almost the same with those obtained in other work-hardened high-Mn TWIP steels, although plasticity of the present steel samples is not so large. ${ }^{[35-39]}$ On the other hand, other multiphase medium-Mn steels commonly exhibit poorer plasticity. ${ }^{[9]}$

It is clearly seen in Figure 9 that all the warm-to-hot rolled steel samples are characterized by almost the same strain hardening behavior upon the subsequent tensile tests at room temperature. A decrease in the rolling temperature just brings the true stress-strain curve to higher stress level. This behavior may indicate on the same strengthening mechanism operating in all steel samples irrespective of previous rolling conditions. Another interesting point to be noted in Figure 9 is the maximal stresses, which are limited by a level of 1200 to $1300 \mathrm{MPa}$, except the initial sample with a coarse grained microstructure after hot rolling at $1423 \mathrm{~K}$. Similar strength limitation has been reported for various high-Mn TWIP steels processed by cold or hot working. ${ }^{[35-39]}$ Such upper limit of the flow stress was considered being associated with a maximal strain 


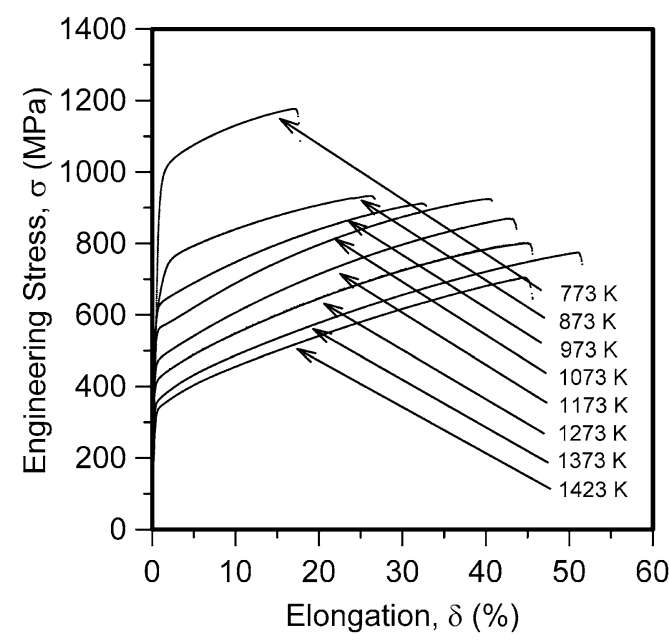

(a)

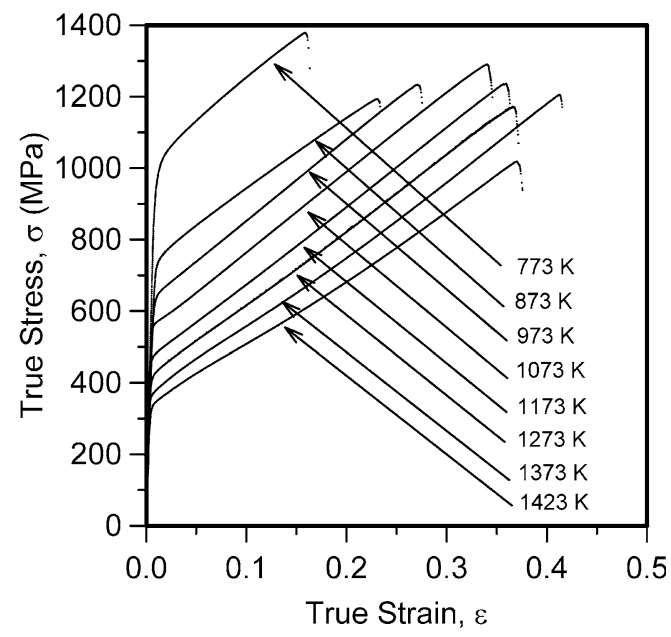

(b)

Fig. 9-Engineering stress-elongation $(a)$ and true stress-strain $(b)$ curves for a medium-Mn steel processed by warm-to-hot rolling at indicated temperatures.

Table II. The Yield Strength $\left(\sigma_{0.2}\right)$, Ultimate Tensile Strength (UTS), and Total Elongation $(\delta)$ of a Medium-Mn Steel Processed by Rolling at Different Temperatures $(T)$

\begin{tabular}{lccc}
\hline$T(\mathrm{~K})$ & $\sigma_{0.2}(\mathrm{MPa})$ & UTS $(\mathrm{MPa})$ & $\delta(\mathrm{Pct})$ \\
\hline 773 & 940 & 1175 & 17 \\
873 & 645 & 930 & 27 \\
973 & 595 & 910 & 33 \\
1073 & 545 & 925 & 40 \\
1173 & 450 & 870 & 43 \\
1273 & 400 & 800 & 45 \\
1373 & 340 & 770 & 51 \\
1423 & 320 & 710 & 46 \\
\hline
\end{tabular}

hardening, which could be assisted by deformation twinning. ${ }^{[35]}$ In this case, further straining beyond the twinning-assisted dislocation storage should inevitably proceed with a localization of plastic flow leading to necking and failure. The high tensile flow stresses recorded for the steel processed by warm rolling at $773 \mathrm{~K}$ may result from deformation twinning, which occurred during warm rolling. Indeed, the grain subdivision by deformation twinning should increase the yield strength upon the subsequent testing. Uniform deformation twinning during warm working may promote homogeneous deformation upon subsequent cold deformation, i.e., tensile test, and provide an additional overall strengthening.

\section{DISCUSSION}

\section{A. Mechanisms of Microstructure Evolution}

The deformation microstructures including the mean grain size and dislocation density that evolved through dynamic recrystallization depend sensitively on the deformation temperature and strain rate. ${ }^{[23,24,40-42]}$ Assuming almost the same strain rate for all warm-to-hot

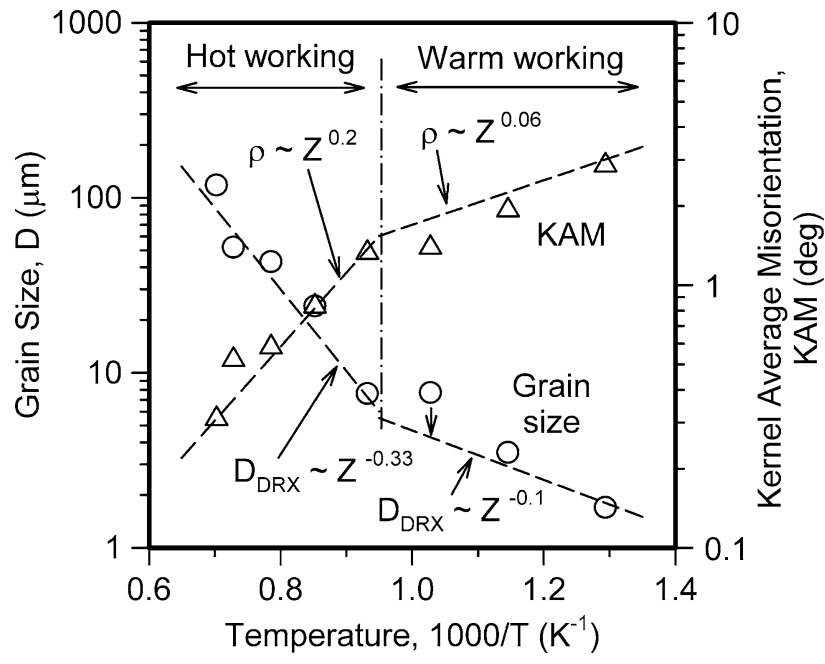

Fig. 10-Effect of rolling temperature on the grain size (circle symbols) and kernel average misorientation, KAM, (triangle symbols) in a medium-Mn steel.

rolling treatments, the structural parameters should solely depend on the rolling temperature. Figure 10 shows the temperature effect on the grain size and kernel average misorientation in the steel samples subjected to warm-to-hot rolling. Note that twin boundaries were omitted while calculating the grain size in Figure 10; and the kernel average misorientation can be referred to here as the dislocation density owing to a direct proportion between these two quantities. ${ }^{[20,43]}$ The dashed lines in Figure 10 indicate some possible relationship between the deformation conditions and microstructural parameters. Under conditions of hot working at temperatures above $1073 \mathrm{~K}$, the mean grain size can be expressed by a power law function of $\mathbf{Z}$ (Zener-Hollomon parameter) with an exponent of approx. -0.33 , which is an average of those of -0.27 to -0.4 reported in other studies on discontinuous dynamic recrystallization (DDRX) in austenite during hot working. ${ }^{[44-47]}$ 
On the other hand, the deformation microstructures evolved during warm deformation are generally associated with continuous dynamic recrystallization $(\mathrm{CDRX}){ }^{[24,25]}$ In this case, the mean grain size can be also expressed by a power law function of $\mathrm{Z}$ with a much smaller exponent of about $-0.1 .^{[45,46]}$ The present data (the range of warm working in Figure 10) display remarkable deviation from the $D_{\mathrm{DRX}} \sim Z^{-0.1}$ line as could be expected for CDRX. This discrepancy is caused by insufficient strain for the development of continuous dynamic recrystallization, which requires much larger strain to make a meaningful contribution to the microstructure evolution. ${ }^{[24,48-50]}$ The present warm rolling with a total reduction of 60 pct leads only to flattening of the original grains and the development of some strain-induced grain boundaries, the number of which is not enough for the grain refinement as expected from the regularities of CDRX. Therefore, dynamic recovery (DRV) can be considered as the main mechanism operating in the steel samples during warm rolling. A decrease in the rolling temperature accelerates the development of strain-induced grain boundaries in austenite ${ }^{[51]}$ Hence, the grain size that developed during warm rolling with a fixed total reduction decreases with a decrease in the rolling temperature. Nevertheless, the temperature dependence of the grain size evolved by warm rolling is weaker than that for hot rolling.

A power law relationship between the dislocation density and the grain size with a grain size exponent of -0.6 , i.e., $\rho \sim D_{\mathrm{DRX}}^{-0.6}$, has been reported for austenitic stainless steels subjected to large strain warm rolling. ${ }^{[0]}$ Thus, setting this relationship in the power law functions between $\mathrm{D}_{\mathrm{DRX}}$ and $\mathrm{Z}$ in Figure 10, the following relationships can be obtained between the dislocation density and deformation conditions: $\rho \sim Z^{0.2}$ and $\rho \sim Z^{0.06}$, for hot and warm working conditions, respectively. It is clearly seen in Figure 10 that the experimental values of KAM (which is directly related to dislocation density) match well the derived power law function in the range of hot working. Similar to the grain size, the kernel average misorientation in Figure 10 does not match perfectly the expected dependence of the dislocation density under warm rolling conditions, although a rough correspondence can be observed. Anyway, the strong temperature dependence of dislocation density during hot working gradually becomes weaker with a decrease in rolling temperature that is probably associated with a rather huge transition region from hot to warm deformation. ${ }^{[40,45]}$ Assuming that the discussed relationships are in force for sufficiently large strains, it can be suggested that a power law function with an exponent of about -0.6 is generally hold between the grain size and the dislocation density in the medium-Mn steel processed by warm-to-hot rolling irrespective of DRV and DDRX operating under different deformation conditions.

The different mechanisms of microstructure evolution during hot and warm rolling lead to different textures developed. The dynamically and/or post-dynamically recrystallized microstructures under conditions of hot rolling are characterized by relatively weak textures, which involve Brass and $\mathrm{S}$ components (Figure 8).
These components are commonly observed in the cold rolled austenite with a low SFE. ${ }^{[32-35]}$ It can be concluded, therefore, that textures in the dynamically and/or post dynamically recrystallized medium-Mn steel are governed by work-hardened grains, which had no chance to recrystallize. An increase in the temperature accelerates the recrystallization processes and weakens the textures. In contrast, warm rolling, which is accompanied by dynamic recovery, leads to strong textures, which enhance with a decrease in the rolling temperature. The deformation textures change from those affected by discontinuous dynamic and/or post dynamic recrystallization to textures, which are typical of cold rolled austenite. The cold rolled textures in austenite have been characterized by a tube with a skeleton line from the Copper component over the $\mathrm{S}$ component to the Brass component. ${ }^{[52]}$ The peak of textural intensity moves along the skeleton line from the Copper to Brass component with a decrease in SFE. Commonly, the Brass and $\mathrm{S}$ components can be expected in the cold rolled austenite with low SFE like the present steel. It is known that SFE in austenite increases with temperature. ${ }^{[28,29]}$ Therefore, the relative intensity of the Brass component increases in the present steel as the warm-rolling temperature decreases (Figure 8).

\section{B. Strengthening}

The grain size effect on the yield strength of the warm-to-hot rolled steel is shown in Figure 11 as Hall-Petch-type plot, i.e.

$$
\sigma_{0.2}=\sigma_{0}+k_{y} D^{-0.5}
$$

where $\sigma_{0}$ is the yield strength of the steel with infinitive grain size and $k_{y}$ is a parameter of the grain boundary strengthening. ${ }^{[53,54]}$ Note here that twin boundaries were counted for the grain size strengthening. The linear

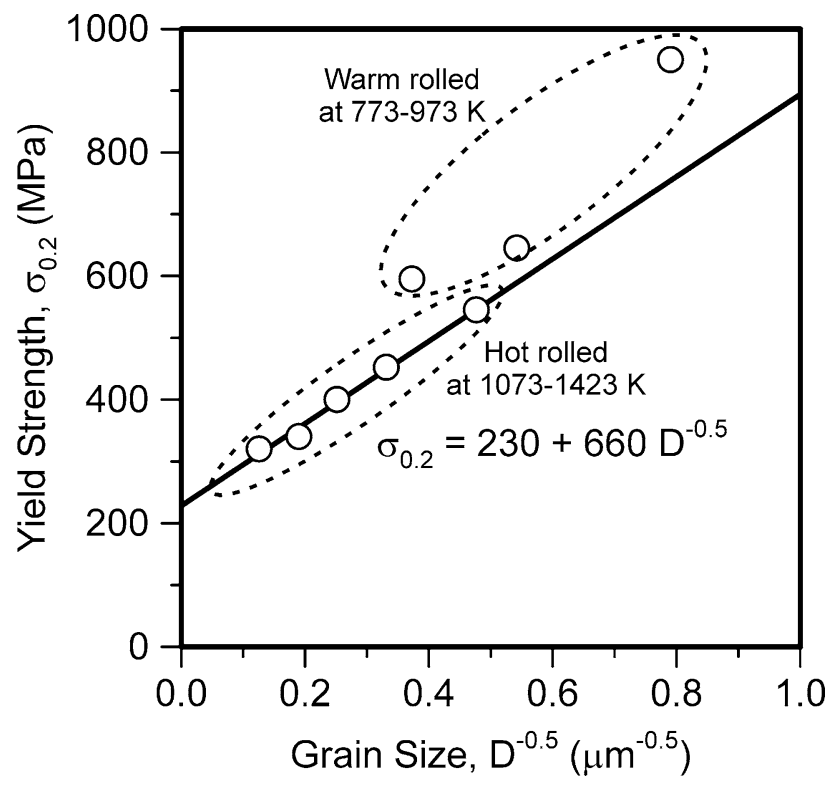

Fig. 11-Hall-Petch-type plot for a medium-Mn steel processed by warm-to-hot rolling. 
relationship between the inverse square root of the grain size and the yield strength is evident for the hot-rolled samples with $\sigma_{0}=230 \mathrm{MPa}$ and $k_{y}=660 \mathrm{MPa} \mu \mathrm{m}^{0.5}$. The present values of $\sigma_{0}$ and $k_{y}$ are somewhat larger than those of 130 to $200 \mathrm{MPa}$ and 350 to $470 \mathrm{MPa} \mu \mathrm{m}^{0.5}$, respectively, reported in other studies on austenitic steels. ${ }^{[40,55-59]}$ Moreover, the yield strengths for the warm rolled samples are well above the straight line corresponding to the hot rolled samples in Figure 11. This strengthening can hardly be explained by the textural changes. An increase in Taylor factor with increasing $\mathrm{P}$ component is compensated by its decrease with increasing Goss component in the warm rolled samples, whereas the change in Brass and $\mathrm{S}$ components do not affect an average Taylor factor of about 3.1 in fcc-metals. ${ }^{[60]}$ Therefore, the relatively large yield strength of the present steel samples as compared to that expected from the grain size strengthening should be attributed to an additional strengthening, which should be taken into account to interpret the structure-property relationship adequately.

The present steel samples subjected to warm or hot rolling are characterized by high internal distortions as proved by the kernel average misorientations in Figure 4. DRV operating during warm working does not provide complete softening that leads to rather high dislocation density, whose quantity increases with a decrease in deformation temperature. ${ }^{[24,39-41]}$ It is interesting to note that hot rolled microstructures that evolve through discontinuous dynamic and post-dynamic recrystallization also include relatively high dislocation densities, which increase with decreasing the deformation temperature. The relatively high internal stresses have been considered as a feature of discontinuous dynamic and post-dynamic recrystallizations. ${ }^{[24,61]}$ The internal stresses in DDRX microstructures that evolve during hot working are attributed to the substructural heterogeneity, which is inherent in the discontinuous dynamic process of microstructure evolution. ${ }^{[24,62]}$ The cyclic character of DDRX implies continuous nucleation and growth of new grains during deformation. The hot worked microstructures, therefore, always contain recrystallization nuclei, growing recrystallized grains, and work-hardened grains. The latter ones should contain high dislocation density, which depends on the rate of dynamic recovery. The development of post-dynamic recrystallization releases partially the internal distortions and decreases the dislocation density. However, post-dynamic recrystallization cannot remove completely the stored dislocations and related internal stresses remaining in growing recrystallized grains.

The yield strength of work-hardened steels and alloys has been expressed by a modified Hall-Petch relationship including a term of substructural (or dislocation) strengthening. ${ }^{[63,64]}$

$$
\sigma_{0.2}=\sigma_{0}+k_{y} D^{-0.5}+\alpha G b \rho^{0.5} .
$$

Here $G$ is the shear modulus and $\alpha$ is a numerical factor ranging from 0.6 to 1.5 as reported in various experimental studies. ${ }^{[25,35,37,39,40,57]}$ The relationship between the yield strength, the grain size, and the dislocation density for the present warm-to-hot rolled steel samples is shown in Figure 12. The dislocation strengthening exhibit almost linear relationship with the grain size strengthening that complicates precise analysis of the structure-property relationship. This linear relationship is governed by the power law functions between the structural parameters, i.e., the grain size and the dislocation density, and the Zener-Hollomon parameter (Figure 10). Similar difficulties have been met in other researches devoted to the same topic. ${ }^{[40,65,66]}$ Nevertheless, the present data in Figure 12 can be well approximated by a plane. The best fit by the least square method $\left(R^{2}=0.95\right)$ is obtained with $\sigma_{0}=65 \mathrm{MPa}$, $k_{y}=420 \mathrm{MPa} \mathrm{m}^{0.5}$, and $\alpha=1.2$.

The extracted grain boundary strengthening factor corresponds well with that found in other similar studies. ${ }^{[40,57,59]}$ On the other hand, the values obtained for $\sigma_{0}$ and $\alpha$ are somewhat different from expected ones. These relatively small $\sigma_{0}$ and large $\alpha$ can be associated with an underestimation of the dislocation density. In fact, the dislocation density calculated by using KAM depends significantly on the OIM step size, the correct value of which depends, in turn, on the value of dislocation density. ${ }^{[20]}$ The value of underestimation increases with an increase in the OIM step size (for a constant dislocation density) and/or an increase in the real dislocation density (in the case of a constant OIM step size). In the present study, the same OIM step size was selected to simplify the comparative analysis. Since the dislocation density increases with a decrease in deformation temperature, the difference between the dislocation density evolved in the warm-to-hot rolled samples and that calculated from KAM increases with a decrease in the rolling temperature. Assuming a linear function between the difference in the calculated and real dislocation densities irrespective of rolling temperatures, e.g., twofold underestimation, a reasonable value of $\alpha=0.85$ is obtained, although the usage of KAM for dislocation density evaluation should be elaborated in more detail. Nonetheless, the present analysis suggests that the strength of the warm-to-hot rolled austenitic

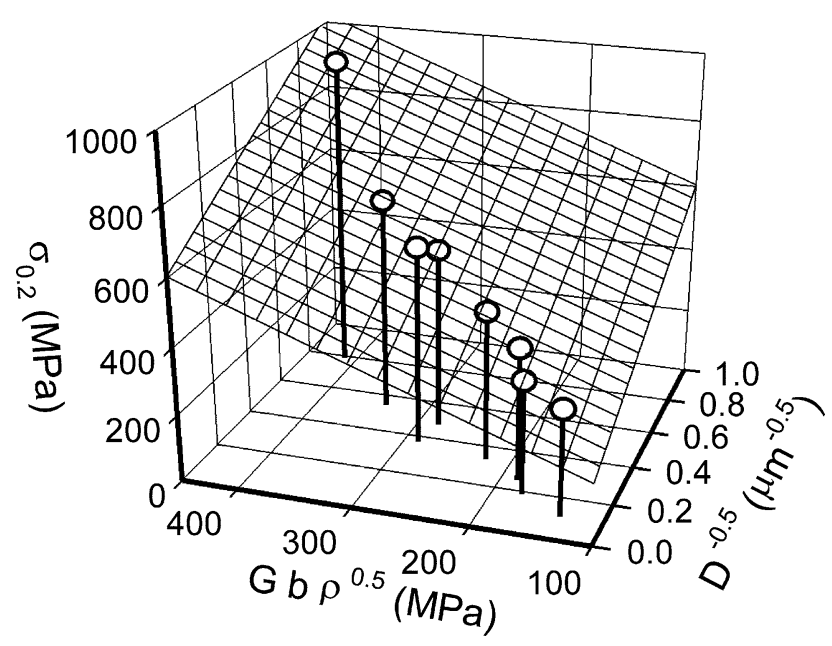

Fig. 12-Relationship between the yield strength, the grain size, and the dislocation density in a warm-to-hot rolled medium-Mn steel. 
steel can be related to the grain size and the dislocation density by using a unique relationship irrespective of the mechanisms of microstructure evolution in a wide range of deformation temperatures.

\section{CONCLUSIONS}

The developed microstructures and their effect on the yield strength of a medium-Mn austenitic steel subjected to warm-to-hot rolling with a total reduction of $60 \mathrm{pct}$ were studied. The main conclusions can be summarized as follows:

1. The deformation microstructures after hot rolling at temperatures of $T \geq 1073 \mathrm{~K}$ consisted of equiaxed grains that resulted from the development of discontinuous dynamic and post-dynamic recrystallization leading to grain refinement down to $4 \mu \mathrm{m}$ with a decrease in temperature. In contrast, during warm rolling at temperatures of $T<1073 \mathrm{~K}$ the structural changes were controlled by dynamic and static recovery. The grain boundary spacing along the normal direction of the warm worked microstructures also decreased with temperature because of increasing the number of strain-induced boundaries and deformation twinning at relatively low temperature.

2. Both the warm and hot rolled microstructures involved large internal distortions, which could be indicative of high dislocation densities. The dislocation density increased in almost ten times with a decrease in rolling temperature from $1373 \mathrm{~K}$ to $773 \mathrm{~K}$ as revealed by means of kernel average misorientation.

3. The deformation textures enhanced with a decrease in rolling temperature; and were characterized by relatively strong Brass, $\mathrm{S}$ and $\mathrm{P}$ components. Among those, the Brass component exhibited the strongest temperature dependence, whereas the $\mathrm{S}$ component displayed the weakest one. The Brass and $\mathrm{S}$ components were predominant in warm and hot rolled samples, respectively.

4. A decrease in rolling temperature resulted in significant strengthening of the steel. The yield strength increased from 340 to $940 \mathrm{MPa}$ as rolling temperature decreased from $1373 \mathrm{~K}$ to $773 \mathrm{~K}$. The yield strength could be expressed by a modified Hall-Petch-type relationship taking into account an additional dislocation strengthening.

\section{ACKNOWLEDGMENTS}

The authors acknowledge with gratitude the financial support received from the Ministry of Education and Science, Russia, under Grant No. 11.3719.2017/ PCh (4.6), and the Deutsche Forschungsgemeinschaft under the Collaborative Research Centre (SFB) Program 761 .

\section{REFERENCES}

1. O. Grässel, L. Krüger, G. Frommeyer, and L.W. Meyer: Int. J. Plast., 2000, vol. 16, pp. 1391-1409.

2. P.S. Kusakin and R.O. Kaibyshev: Rev. Adv. Mater. Sci., 2016, vol. 44 , pp. 326-60.

3. B.C. De Cooman, Y. Estrin, and S.K. Kim: Acta Mater., 2018, vol. 142 , pp. $283-362$.

4. A. Saeed-Akbari, L. Mosecker, A. Schwedt, and W. Bleck: Metall. Mater. Trans. A, 2012, vol. 43A, pp. 1688-1704.

5. J.-K. Kim and B.C. De Cooman: Mater. Sci. Eng. A, 2016, vol. A676, pp. 216-31.

6. Y. Kim, N. Kang, Y. Park, I. Choi, G. Kim, S. Kim, and K. Cho: J. Korean Inst. Met. Mater., 2008, vol. 46, pp. 780-87.

7. I. Nikulin, T. Sawaguchi, and K. Tsuzaki: Mater. Sci. Eng. A, 2013, vol. A587, pp. 192-200.

8. M. Koyama, Y. Yamamura, R. Che, T. Sawaguchi, K. Tsuzaki, and H. Noguchi: Int. J. Fatigue, 2017, vol. 94, pp. 1-5.

9. Y.-K. Lee and J. Han: Mater. Sci. Technol., 2015, vol. 31, pp. 843-56.

10. J. Han, A. Kwiatkowski da Silva, D. Ponge, D. Raabe, S.-M. Lee, Y.-K. Lee, S.-I. Lee, and B. Hwang: Acta Mater., 2017, vol. 122, pp. 199-206.

11. Y. Li, W. Li, N. Min, W. Liu, and X. Jin: Acta Mater., 2017, vol. 139 , pp. $96-108$.

12. K. Li, V.S.Y. Injeti, R.D.K. Misra, Z.H. Cai, and H. Ding: Mater. Sci. Eng. A, 2018, vol. A711, pp. 515-23.

13. X.Y. Qi, L.X. Du, J. Hu, and R.D.K. Misra: Mater. Sci. Eng. A, 2018, vol. A718, pp. 477-82.

14. E.J. Seo, J.K. Kim, L. Cho, J. Mola, C.Y. Oh, and B.C. De Cooman: Acta Mater., 2017, vol. 135, pp. 112-23.

15. K. Steineder, D. Krizan, R. Schneider, C. Beal, and C. Sommitsch: Acta Mater., 2017, vol. 139, pp. 39-50.

16. T. Furukawa, H. Huang, and O. Matsumura: Mater. Sci. Technol., 1994, vol. 10, pp. 964-70.

17. P.J. Gibbs, E. De Moor, M.J. Merwin, B. Clausen, J.G. Speer, and D.K. Matlock: Metall. Mater. Trans. A, 2011, vol. 42A, pp. 3691-3702.

18. S. Lee and B.C. De Cooman: Metall. Mater. Trans. A, 2013, vol. 44A, pp. 5018-24.

19. V. Torganchuk, D.A. Molodov, A. Belyakov, and R. Kaibyshev: Defect Diffus. Forum, 2018, vol. 385, pp. 308-13.

20. M. Calcagnotto, D. Ponge, E. Demir, and D. Raabe: Mater. Sci. Eng. A, 2010, vol. A527, pp. 2738-46.

21. F. Bachmann, R. Hielscher, and H. Schaeben: Solid State Phenom., 2010, vol. 160, pp. 63-68.

22. H.J. McQueen and J.J. Jonas: in Treatise on Materials Science and Technology, R.J. Arsenault, ed., Academic Press, New York, 1975, vol. 6, pp. 394-493.

23. T. Sakai and J.J. Jonas: Acta Metall., 1984, vol. 32, pp. 189-209.

24. T. Sakai, A. Belyakov, R. Kaibyshev, H. Miura, and J.J. Jonas: Prog. Mater. Sci., 2014, vol. 60, pp. 130-207.

25. M. Tikhonova, R. Kaibyshev, and A. Belyakov: Adv. Eng. Mater., 2018, vol. 20, p. 1700960.

26. L. Rémy: Metall. Trans. A, 1981, vol. 12A, pp. 387-408.

27. E.I. Galindo-Nava and P.E.J. Rivera-Díaz-del-Castillo: Acta Mater., 2017, vol. 128, pp. 120-34.

28. P.C.J. Gallagher: Metall. Trans., 1970, vol. 1, pp. 2429-61.

29. R.M. Latanision and A.W. Ruff: Metall. Trans., 1971, vol. 2, pp. 505-09.

30. F.J. Humphreys and M. Hatherly: Recrystallization and Related Annealing Phenomena, 2nd ed., Elsevier Science, New York, 2004.

31. J.K. Mackenzie: Biometrika, 1958, vol. 45, pp. 229-40.

32. Y. Lü, D.A. Molodov, and G. Gottstein: ISIJ Int., 2011, vol. 51, pp. 812-17.

33. C. Haase, S.G. Chowdhury, L.A. Barrales-Mora, D.A. Molodov, and G. Gottstein: Metall. Mater. Trans. A, 2013, vol. 44A, pp. 911-22.

34. C. Haase, T. Ingendahl, O. Güvenç, M. Bambach, W. Bleck, D.A. Molodov, and L.A. Barrales-Mora: Mater. Sci. Eng. A, 2016, vol. A649, pp. 74-84.

35. Z. Yanushkevich, A. Belyakov, C. Haase, D.A. Molodov, and R. Kaibyshev: Mater. Sci. Eng. A, 2016, vol. A651, pp. 763-73. 
36. M. Klimova, G. Dyakonov, S. Zherebtsov, G. Salishchev, and D. Molodov: IOP Conf. Series: Mater. Sci. Eng., 2014, vol. 63, art. No. 012157.

37. P. Kusakin, K. Tsuzaki, D.A. Molodov, R. Kaibyshev, and A. Belyakov: Metall. Mater. Trans. A, 2016, vol. 47A, pp. 5704-08.

38. C. Haase, C. Zehnder, T. Ingendahl, A. Bikar, F. Tang, B. Hallstedt, W. Hu, W. Bleck, and D.A. Molodov: Acta Mater., 2017, vol. 122, pp. $332-43$

39. V. Torganchuk, A. Belyakov, and R. Kaibyshev: Mater. Sci. Eng. $A$, 2017, vol. A708, pp. 110-17.

40. Z. Yanushkevich, S.V. Dobatkin, A. Belyakov, and R. Kaibyshev: Acta Mater., 2017, vol. 136, pp. 39-48.

41. Y.-X. Liu, Y.C. Lin, and Y. Zhou: Mater. Sci. Eng. A, 2017, vol. A691, pp. 88-99.

42. D.-G. He, Y.C. Lin, J. Huang, and Y. Tang: Adv. Eng. Mater., 2018, vol. 20, p. 1800129.

43. P. Kusakin, A. Kalinenko, K. Tsuzaki, A. Belyakov, and R. Kaibyshev: Kovove Materialy, 2017, vol. 55, pp. 161-67.

44. T. Maki, K. Akasaka, K. Okuno, and I. Tamura: Trans. Iron Steel Inst. Jpn., 1982, vol. 22, pp. 253-61.

45. M. Tikhonova, A. Belyakov, and R. Kaibyshev: Mater. Sci. Eng. A, 2013, vol. A564, pp. 413-22.

46. Z. Yanushkevich, A. Lugovskaya, A. Belyakov, and R. Kaibyshev: Mater. Sci. Eng. A, 2016, vol. A667, pp. 279-85.

47. M. Tikhonova, P. Dolzhenko, R. Kaibyshev, and A. Belyakov: Metals, 2016, vol. 6, p. 268.

48. S. Gourdet and F. Montheillet: Acta Mater., 2003, vol. 51, pp. 2685-99.

49. N. Dudova, A. Belyakov, T. Sakai, and R. Kaibyshev: Acta Mater., 2010, vol. 58, pp. 3624-32.

50. L.S. Toth, Y. Estrin, R. Lapovok, and C. Gu: Acta Mater., 2010, vol. 58, pp. 1782-94.
51. Z. Yanushkevich, A. Belyakov, and R. Kaibyshev: Acta Mater., 2015, vol. 82, pp. 244-54.

52. J. Hirsch and K. Lucke: Acta Met. Mater., 1988, vol. 36, pp. 2863-82.

53. E.O. Hall: Proc. Phys. Soc. B, 1951, vol. 64, pp. 742-47.

54. N.J. Petch: J. Iron Steel Inst., 1953, vol. 174, pp. 25-28.

55. C.M. Young and O.D. Sherby: J. Iron Steel Inst., 1973, vol. 211, pp. 640-47.

56. P. Kusakin, A. Belyakov, R. Kaibyshev, and D. Molodov: Mater. Sci. Forum, 2016, vols. 838-839, pp. 392-97.

57. Z. Yanushkevich, A. Belyakov, R. Kaibyshev, C. Haase, and D.A. Molodov: Mater. Charact., 2016, vol. 112, pp. 180-87.

58. M. Odnobokova, A. Belyakov, N. Enikeev, D.A. Molodov, and R. Kaibyshev: Mater. Sci. Eng. A, 2017, vol. A689, pp. $370-83$.

59. A. Kalinenko, P. Kusakin, A. Belyakov, R. Kaibyshev, and D.A. Molodov: Metals, 2017, vol. 7, p. 571.

60. J. Gil Sevillano, P. van Houtte, and E. Aernoudt: Prog. Mater. Sci., 1981, vol. 25, pp. 69-412.

61. T. Sakai: J. Mater. Process. Technol., 1995, vol. 53, pp. 349-61.

62. T. Sakai and M. Ohashi: Mater. Sci. Technol., 1990, vol. 6, pp. 1251-57.

63. Y. Estrin, L.S. Toth, A. Molinari, and Y. Brechet: Acta Mater., 1998, vol. 46, pp. 5509-22.

64. N. Hansen: Scr. Mater., 2004, vol. 51, pp. 801-06.

65. I. Shakhova, A. Belyakov, Z. Yanushkevich, K. Tsuzaki, and R. Kaibyshev: ISIJ Int., 2016, vol. 56, pp. 1289-96.

66. M.J. Starink: Mater. Sci. Eng. A, 2017, vol. A705, pp. 42-45.

Publisher's Note Springer Nature remains neutral with regard to jurisdictional claims in published maps and institutional affiliations. 